\title{
COVID-19 pandemic evolution in the Brazilian Indigenous population
}

\author{
Matheus Ferreira Mendes ${ }^{1,2}$ (D) $\cdot$ Letícia Rogini Pereira $^{1,2}$ (D) $\cdot$ Tainá Momesso Lima $^{1,2}$ (D) $\cdot$ Vitória Franchini Melani $^{1,2}$ (D) \\ Camila Vantini Capasso Palamim ${ }^{1,2}$ (D) $\cdot$ Matheus Negri Boschiero ${ }^{1,2}$ (D) Fernando Augusto Lima Marson ${ }^{1,2}$ (D)
}

Received: 24 February 2021 / Revised: 20 March 2021 / Accepted: 22 March 2021 / Published online: 29 March 2021

(C) W. Montague Cobb-NMA Health Institute 2021

\begin{abstract}
Introduction The COVID-19 pandemic has affected several neglected populations such as the Indigenous peoples, which have suffered a high impact from the pandemic.

Objectives To analyze the impact on the health and disease process according to the COVID-19 evolution in the Brazilian Indigenous population.

Methods Data was collected from press releases by the Health Ministry and a descriptive analysis of the numbers of Indigenous individuals infected with the SARS-CoV-2 in Brazil was carried out.

Results In February 2021, there were 41,855 confirmed cases of Indigenous individuals infected by the SARS-CoV-2, including 4,387 active cases, 36,809 recovered cases, and 549 deaths. The Brazilian Indigenous population is distributed in over 300 ethnic groups and, due to the high number of deaths by the COVID-19, many of these groups are endangered. The elderly are the most affected age group, and they play a fundamental role among the Indigenous population for transmitting their customs mainly orally. Indigenous populations do not have proper access to transport to specialized health centers, since many areas are inaccessible and other cases require air or river transportation, which many times results in late assistance. When managing the COVID-19, it is important to emphasize the need for social isolation to prevent the virus from spreading among the Indigenous groups, mainly due to their contact with other ethnic groups represented by missionaries, hunters, and wood explorers, among others.

Conclusion The adoption of practices that can reduce the virus transmission among the Indigenous population and provide them with better access to treatment, mainly for the elderly, must be prioritized in Brazil.
\end{abstract}

Keywords Indigenous population $\cdot$ Pandemic $\cdot$ Social isolation $\cdot$ SARS-CoV-2 $\cdot$ COVID-19 $\cdot$ Vulnerable group

Matheus Ferreira Mendes, Letícia Rogini Pereira, Tainá Momesso Lima, Vitória Franchini Melani, Camila Vantini Capasso Palamim, Matheus Negri Boschiero and Fernando Augusto Lima Marson contributed equally to this work.

Fernando Augusto Lima Marson

fernandolimamarson@hotmail.com; fernando.marson@usf.edu.br

Matheus Ferreira Mendes

matheus.ferreira@mail.usf.edu.br

Letícia Rogini Pereira

leticiarogini@gmail.com

Tainá Momesso Lima

tainamomessolima65@gmail.com

Vitória Franchini Melani

vivismelani@gmail.com
Camila Vantini Capasso Palamim

cvcpalamim@gmail.com

Matheus Negri Boschiero

negri.matheus@bol.com.br

1 Laboratory of Cell and Molecular Tumor Biology and Bioactive Compounds, São Francisco University, Avenida São Francisco de Assis, 218. Jardim São José, Bragança Paulista, São Paulo 12916-900, Brazil

2 Laboratory of Human and Medical Genetics, São Francisco University, Avenida São Francisco de Assis, 218. Jardim São José, Bragança Paulista, São Paulo 12916-900, Brazil 


\section{Introduction}

On March 11, 2020, the World Health Organization declared that the COVID-19 could be characterized as a pandemic due to the spread of the coronavirus disease 2019 (COVID-19) [1] caused by the severe acute respiratory syndrome coronavirus 2 (SARS-CoV-2) [2]. Facing this scenery, the analysis of the health and disease processes in different social contexts became relevant for the COVID-19 control, mainly when considering the great territorial extension, the social and demographic aspects, and the populational heterogeneity in Brazil. Thus, a detailed analysis of each of the groups that form the Brazilian population is essential, mainly those groups that present higher degree of vulnerability to pandemic situations, such as the Indigenous peoples (Fig.1).

Indigenous peoples, despite representing $0.4 \%$ of the Brazilian population, according to the census carried out by the Brazilian Institute of Geography and Statistics (IBGE) in 2000, constitute a cultural heritage that comprises 305 ethnic groups and 274 languages [3]. The struggle for the recognition of Indigenous rights is historical and continues until nowadays. The recognition of the Indigenous citizenship and guarantee of respect to their customs and traditions, health, rights to the lands traditionally occupied by indigenous tribes was only enacted in 1988 with the approval of the Brazilian Federal Constitution. Consequently, an increase in their political representativeness occurred along with achievements in the social area that resulted in a process of ethnic emergence with a $177 \%$ indigenous population growth in a period of 20 years [4].

The indigenous peoples' achievements enabled better integration between this group and the rest of the Brazilian population, so that many Indigenous individuals live in the urban zone currently. However, their proximity with other ethnic groups was one of the factors that favored the spread of the SARS-CoV-2, and the first case of infection was associated to the contact with an infected medical doctor [5]. At the same time, wood exploration, deforestation of environmental preservation areas, and illegal mining increased the risk of these native people to be infected with the SARS-CoV-2 and to get other infectious diseases [5-7]. Also, despite the Indigenous peoples having constitutional rights, in practice they are not applicable, contrariwise, they are often violated by the Brazilian government through the adoption of antienvironmental measures that resulted in the highest rates of burning in the Amazon forest in the last 20 years. The federal government attitudes regarding environmental prompted the Brazil forests to devastation being observed a record of loss of vegetation cover, mainly on Amazon lands [8]. The neglection of the federal government in protecting Indigenous peoples against COVID-19 and its lands was described in the literature demonstrating a high impact of the COVID-19 pandemic on this population due the absence of better federal government attitudes $[8,9]$.
In contrast, state actions related to the Indigenous health started in 1999 through the division of their territory into Indigenous Health Special Districts (DSEIs, Brazilian acronym for Distritos de Saúde Especial Indígena), with the purpose of offering specific health measures to the local groups. Indigenous Health Units and the Indigenous Health Houses (CASAI, Brazilian acronym for Casas de Saúde Indígena) were implemented in the DSEIs, which were responsible for the medical assistance of higher complexity. Another measure adopted was the qualification of Indigenous individuals to work as health agents. The measures showed a beneficial effect on this population health resulting in a reduction in the mortality rate from $7.1 \%$ to $4.77 \%$, in the period between 2000 and 2005 [10]. Despite the improvement in the Indigenous assistance, many individuals still refuse their own or the transportation of members of their families to more specialized health centers; for this reason, optimized medical assistance that respect the cultural divergences and traditions of those peoples is required [11]. Concomitantly, equipment, materials, and professionals are scarce, and the difficult access to the health units hampers such assistance [12].

The COVID-19 pandemic is a global challenge and a threat to the Indigenous culture, since many native customs started to be practiced only by the elderly and might get lost after these older individuals' deaths - this is the age group most affected by the COVID-19 [13]. According to all the limitations related to the Brazilian indigenous health, it becomes relevant to analyze how this group has faced the pandemic in a prospective study. Therefore, this study aimed to verify the evolution of the COVID-19 impact on Indigenous peoples, regarding cultural aspects and their survival at a national level based on the epidemiological notes published by the Brazilian Health Ministry. In addition, a descriptive analysis of the number of cases of Indigenous individuals infected with the SARS-CoV-2 in Brazil is provided.

\section{Methods}

A prospective data survey was carried out based on the epidemiological notes released by the Brazilian Health Ministry [14]. The full text of all notes published were read and evaluated by the researchers responsible for this study. Notes whose content was about government actions such as the distribution of individual protection equipment (IPE) and clarifying notes by the Health Ministry regarding facts occurred were excluded, while those specifically related to the COVID-19 pandemic progression in the Indigenous population were included.

The data collected from the notes were (i) name of the DSEI of origin of the Indigenous patient; (ii) ethnic group that the Indigenous patient belonged according to the National Indian Foundation (FUNAI, Brazilian acronym for Fundação Nacional do Índio); (iii) age (years); (iv) 
Fig. 1 Indigenous painting on canvas. Art by the Brazilian painter Elvis da Silva and purchased by the corresponding author in 2015

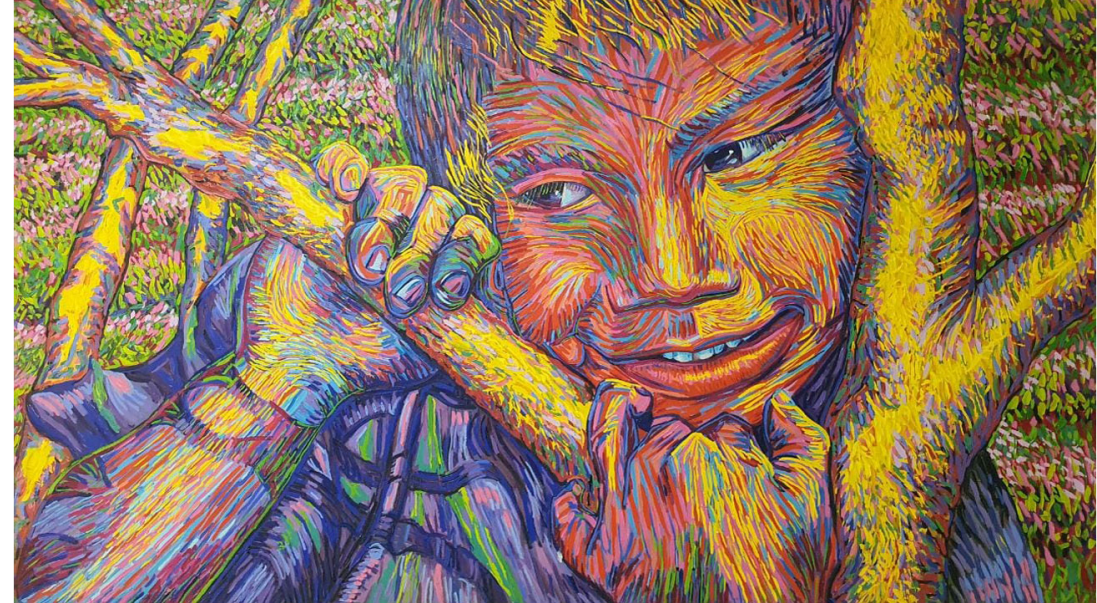

comorbidity; and (v) social status within the Indigenous community. In the case of death, the date was recorded. Other information used, whenever available, was the disease transmission history and how many people might have been infected with SARS-Co-V-2 from one confirmed case in the community.

The instructions to the health units on the treatment and monitoring of Indigenous patients were given by the Health Ministry. The DSEI is a management unit decentralized from the Indigenous Health Attention Subsystem, so that the health service becomes guided to a certain ethnic-cultural group. The DSEIs are structured in Indigenous Basic Health Units (simple infrastructure destined to basic procedures and patients' follow-up), base centers (structure with a multidisciplinary team and that might be located inside the indigenous community or in reference municipalities), and the CASAIs (places of support to the indigenous population, for example, to provide accommodation to the Indigenous patient and their families during the treatment, which includes assistance to patients after hospital discharge).

The Indigenous Health System provided information such as the total number of COVID-19-suspected cases (Indigenous individuals with acute respiratory symptoms that left the tribe or that had contact with another suspected or confirmed case in the last 14 days), COVID-19-confirmed cases (cases were described as confirmed after laboratory analysis and by clinical criteria), COVID-19-dismissed cases (suspected case with negative laboratory result for the SARSCoV-2 identification), COVID-19-infected cases (confirmed cases with active infection), clinical cure (cases confirmed that spent 10 days in home isolation, as of the start of the symptoms, and that were asymptomatic for $24 \mathrm{~h}$ ), and number of deaths (cases presenting death as a result of the SARS-CoV-2 infection and its complications). Finally, the 1 st phase of the COVID-19 vaccination program in Brazil considers the Indigenous population living on indigenous land as a priority group.

\section{Results}

\section{Description of official notes by the Health Ministry in relation to the COVID-19 evolution in the Indigenous population}

Out of the 79 notes evaluated, 70 were selected, which included specific reports of Indigenous cases aiming to evaluate parameters such as death, comorbidity presence, social function, and other problems faced by those individuals. The remaining 9 notes were excluded for only describing the contamination of professionals related to the DSEIs (medical doctors and other health professionals, DSEIs coordinators, 51 Indigenous individuals) without specifying details. There was also a note reporting the death of a FUNAI worker.

Table 1 presents the content of the notes published by the Health Ministry. According to the age range of those infected:

( $<1$ year old) two releases described death; malnutrition was reported in one of the cases;

(Individuals between 10 and 19 years old) six releases; five of them reported death. One of these patients presented tuberculosis and congenital cardiopathy;

(Individuals between 20 and 29 years old) three releases; none of them reported death;

(Individuals between 30 and 39 years old) four releases; three of them reported the death of the Indigenous patient, including a tribe chief. Another of these patients who died was diagnosed with diabetes mellitus (DM) and suspected cancer, and the other was a nurse from the Indigenous Health Multidisciplinary Team who was diagnosed with asthma;

(Individuals between 40 and 49 years old) four releases and all of them reported death. One of them was a tribe advisor and had influence in the local leadership. The comorbidities found were autoimmune hemolytic anemia (one patient); chronic kidney disease (one patient); obesity and systemic arterial hypertension (SAH) (one patient); 


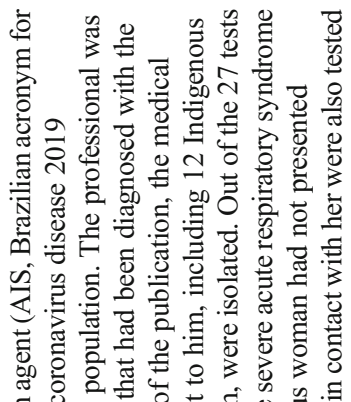

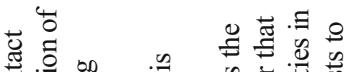
ョ节 要恶

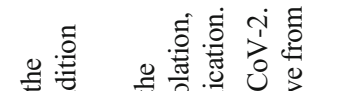

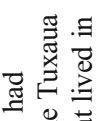

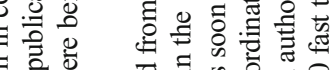

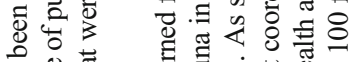

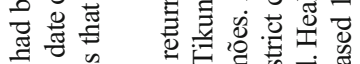

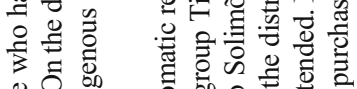

总

客.

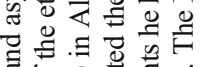

\&

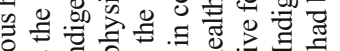

焉

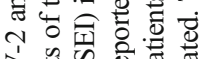

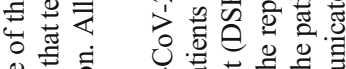

荡

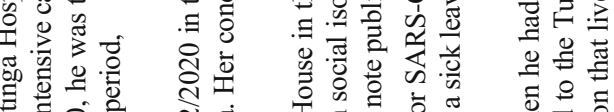

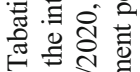

웡 융

तิ

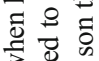

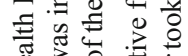

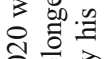

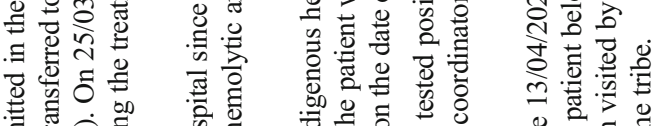

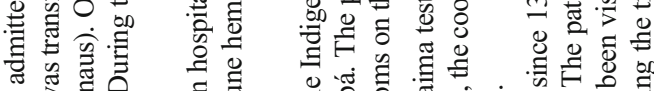

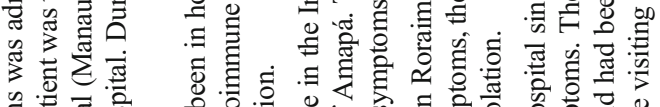

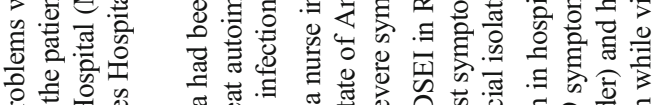

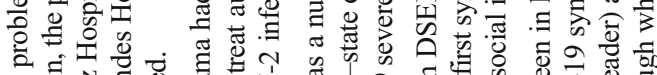

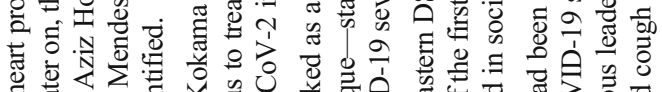

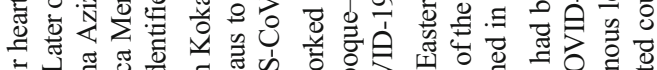

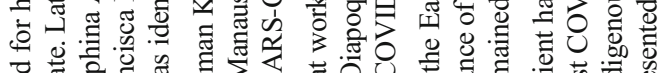

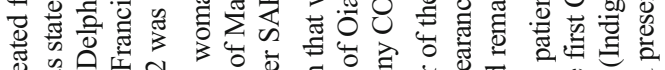

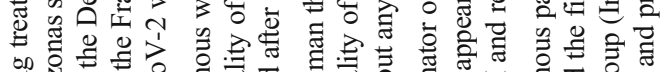
on $\begin{gathered}0 \\ \text { o } \\ 0\end{gathered}$

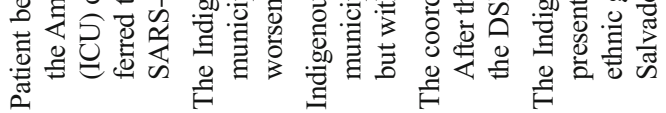

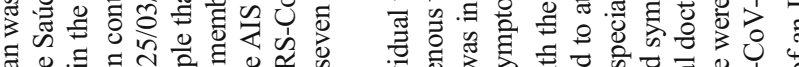

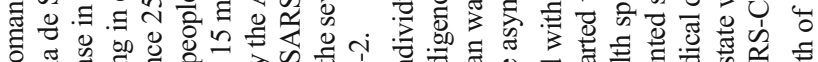

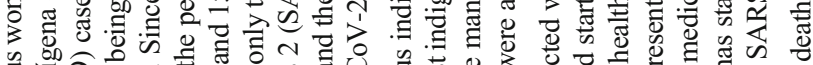

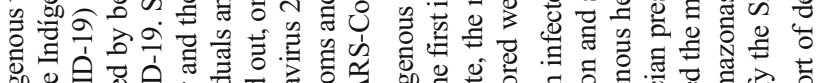

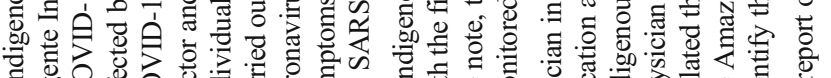

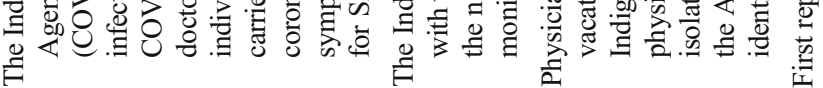

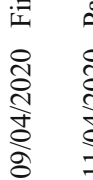

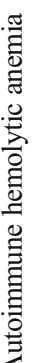

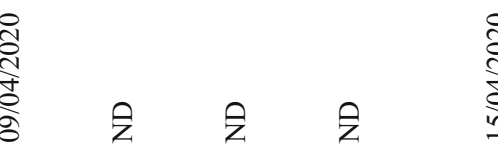

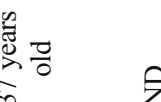

之奇

之合 至

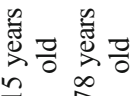

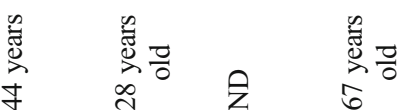

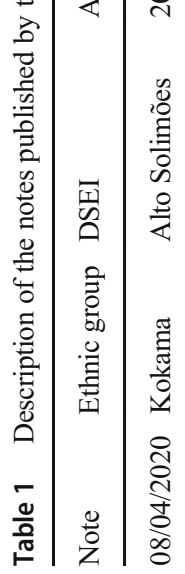

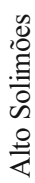

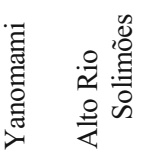

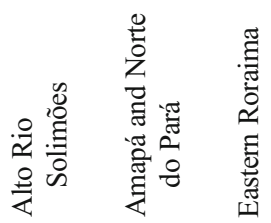

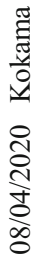

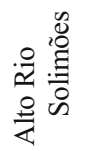

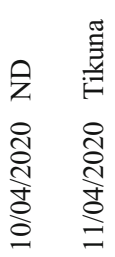

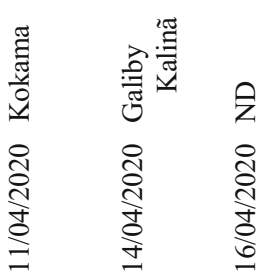

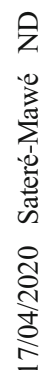

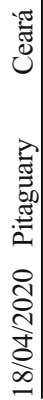




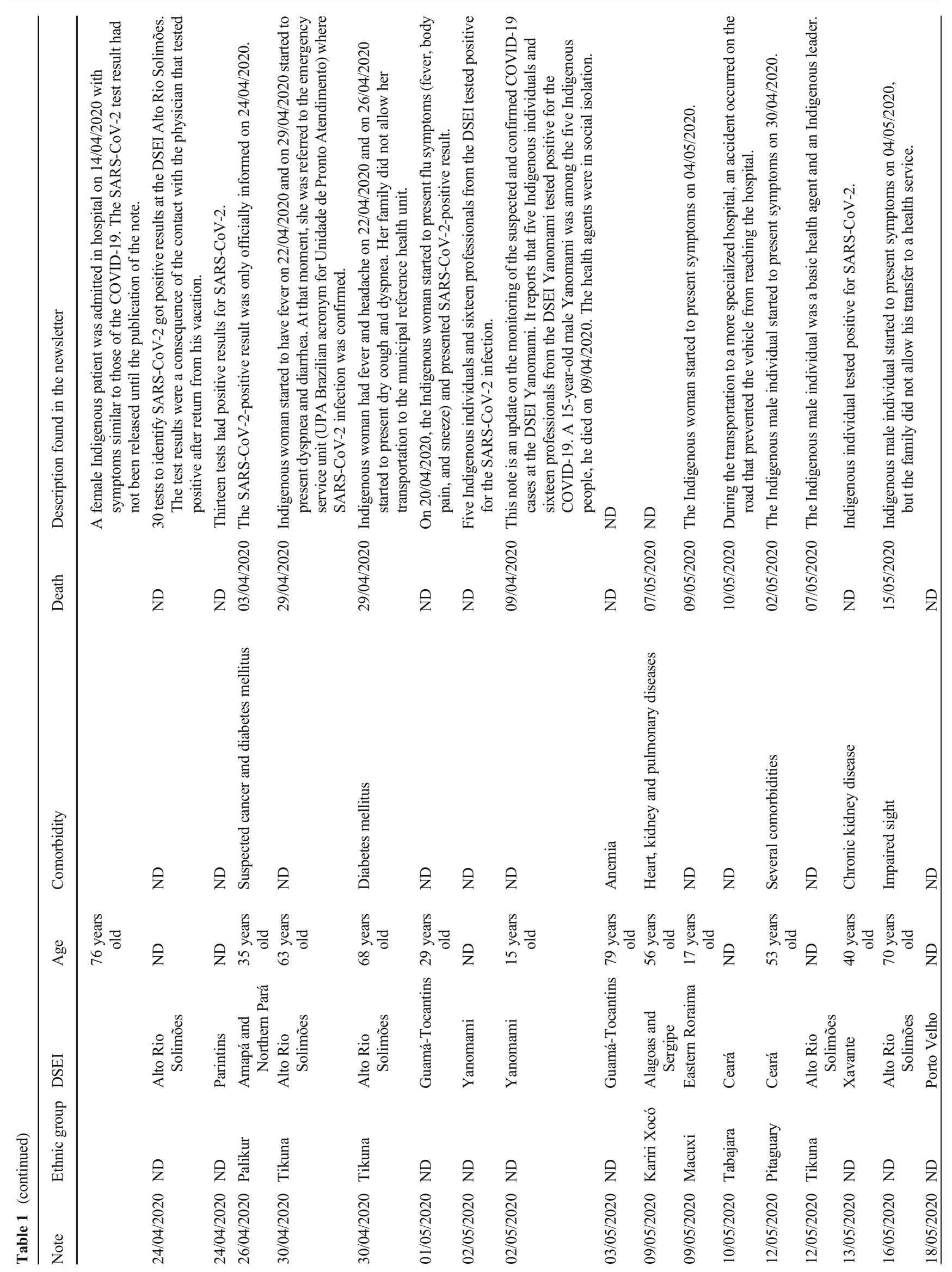




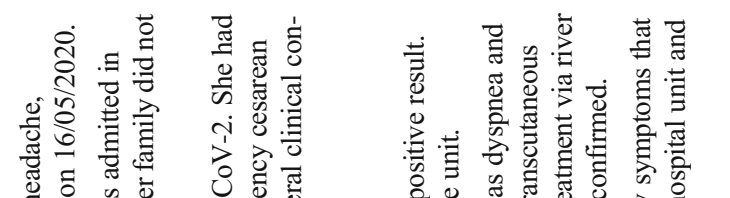
ฮี

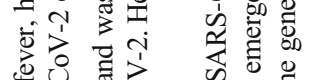

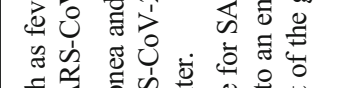

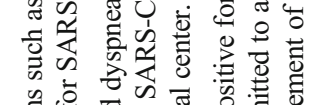

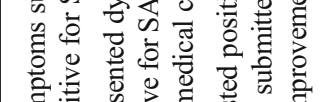

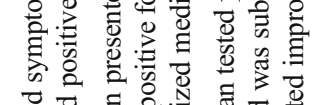

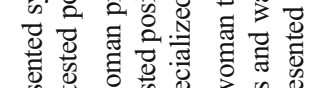
足苟

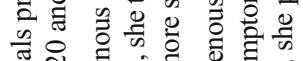
चั

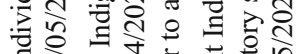

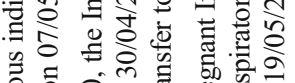

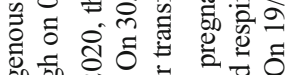

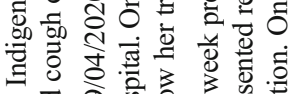

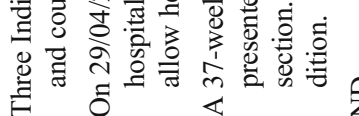

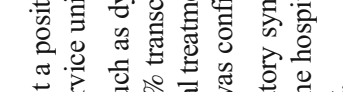

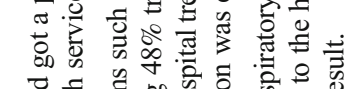

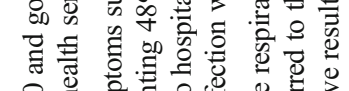

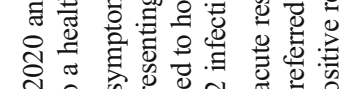

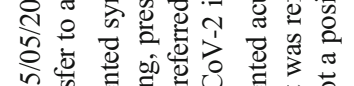

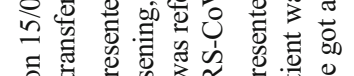
हี

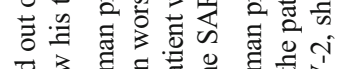

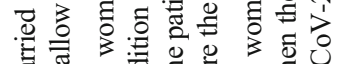

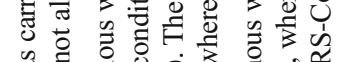

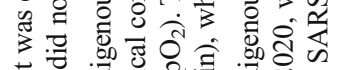

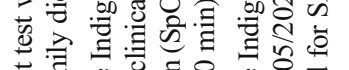

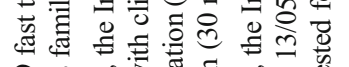

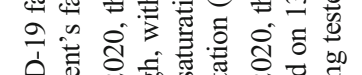

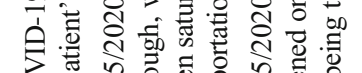
ว 气ัे

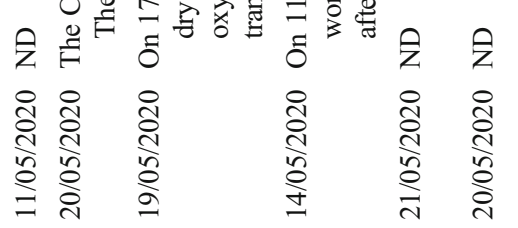

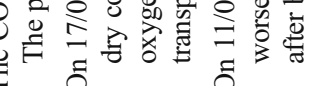

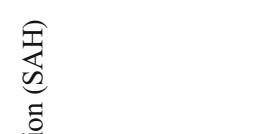
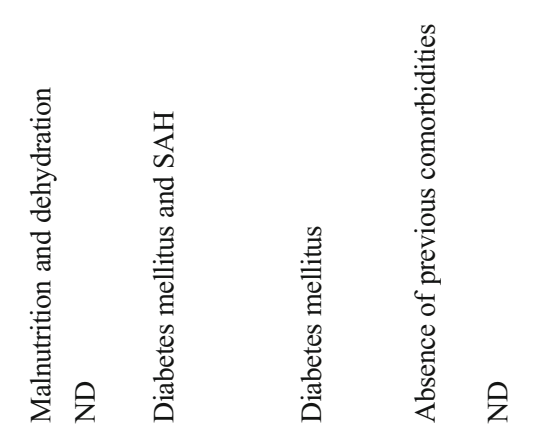

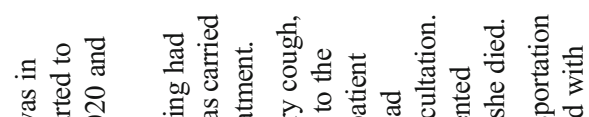

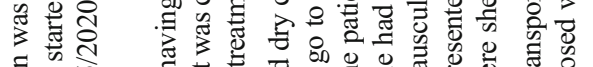

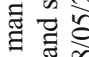

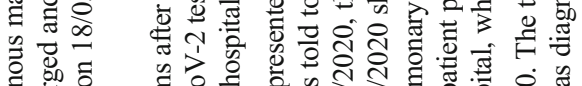

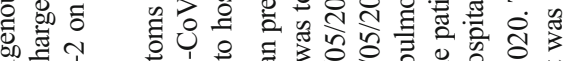

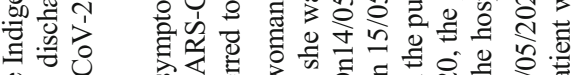

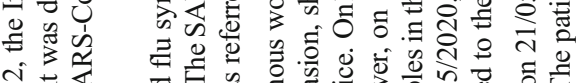

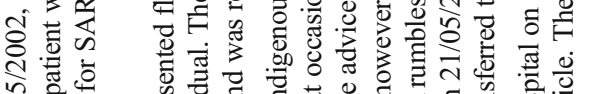

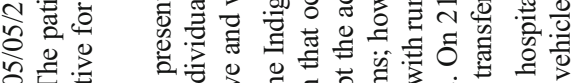

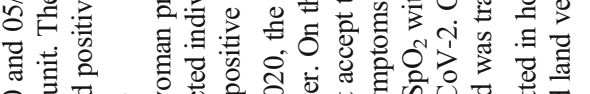

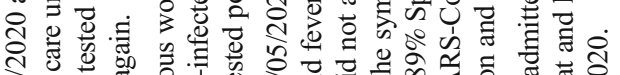

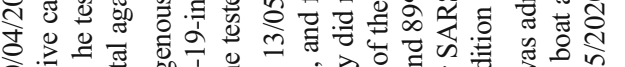

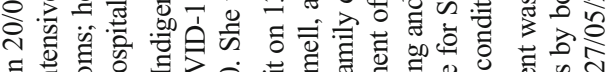

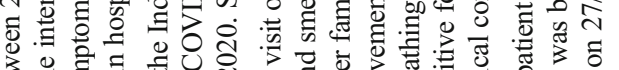

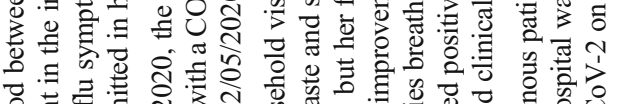

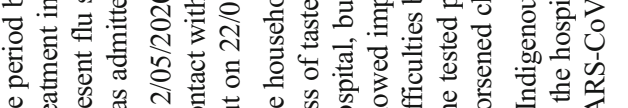

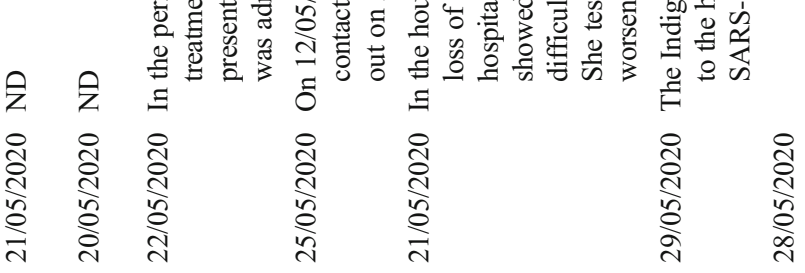




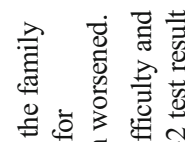

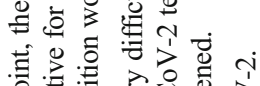

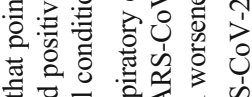

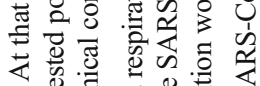

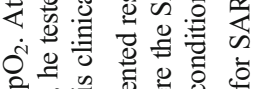

की

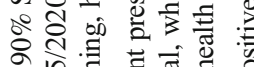

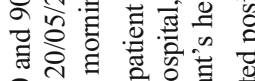

॰

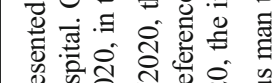

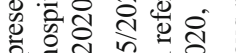

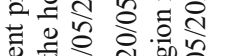

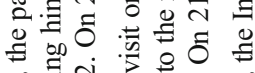

จิ

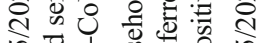

ऊิ चे

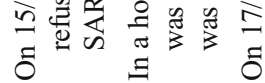

芩

崩

言

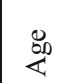

牙

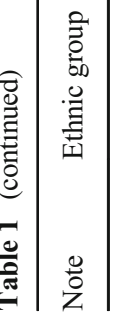

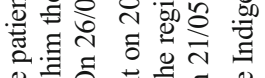

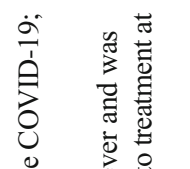

离

品

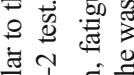

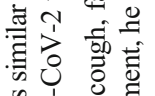

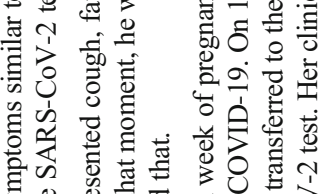

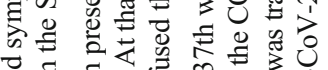

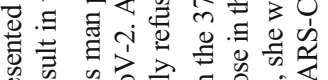

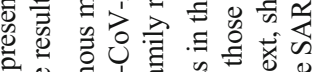

20

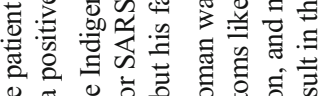

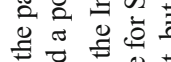

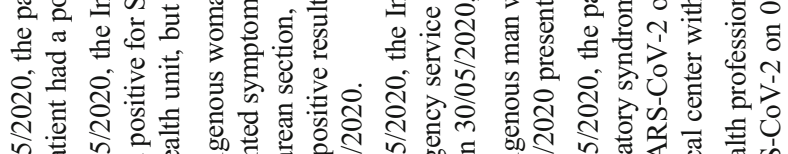

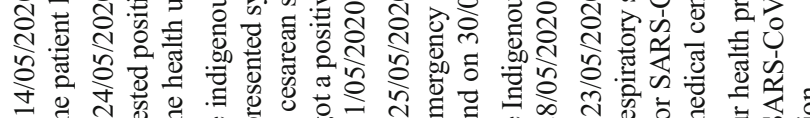

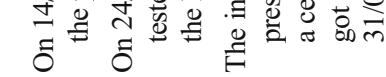

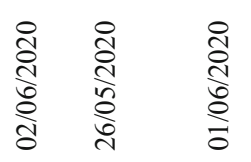

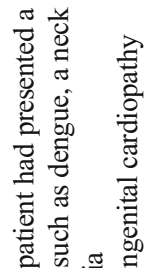

旅

焉

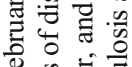

乙

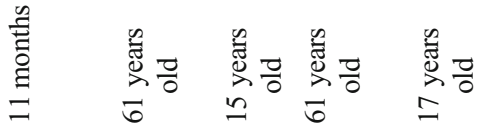

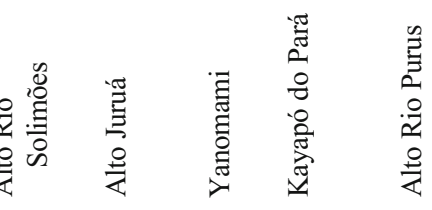

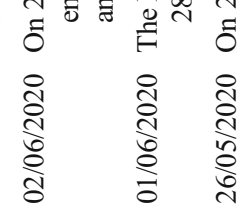

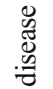

荧

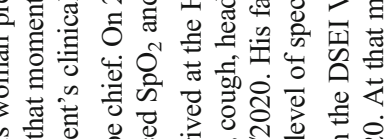

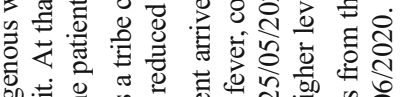

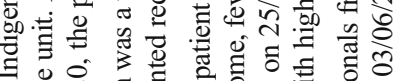

चै

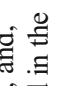

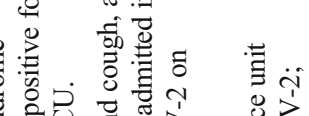

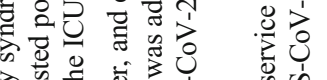

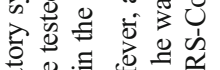

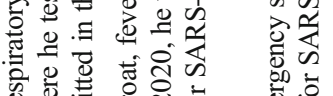

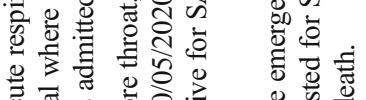

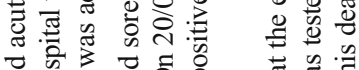

च

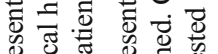

跣

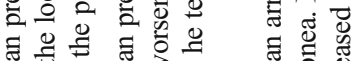

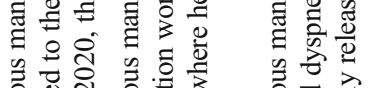

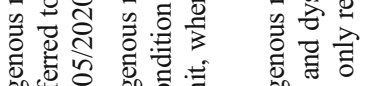

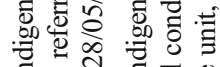

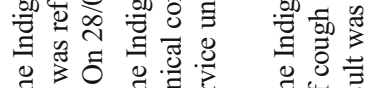

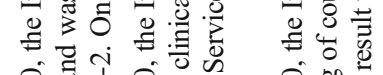

ปิ่

ऊิ

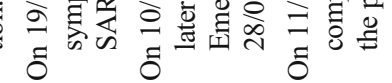




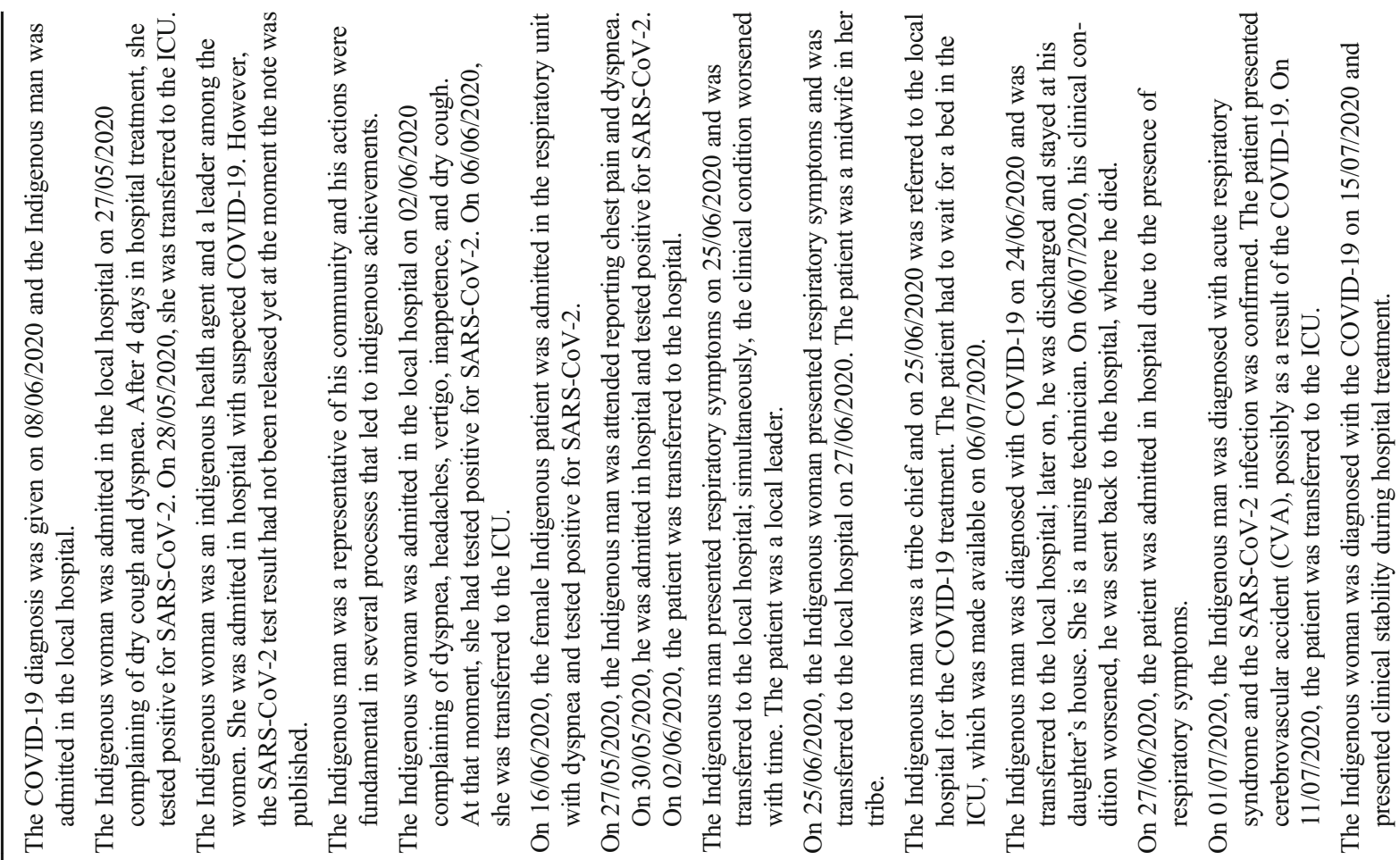

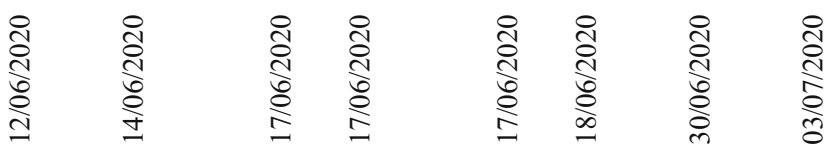

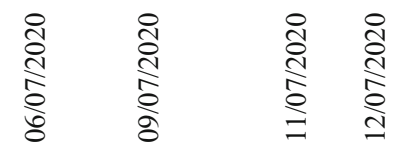

之

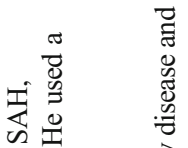

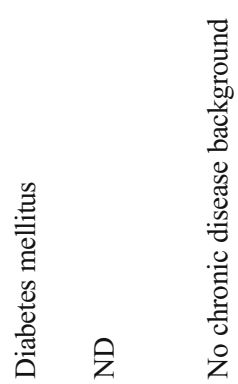

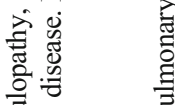

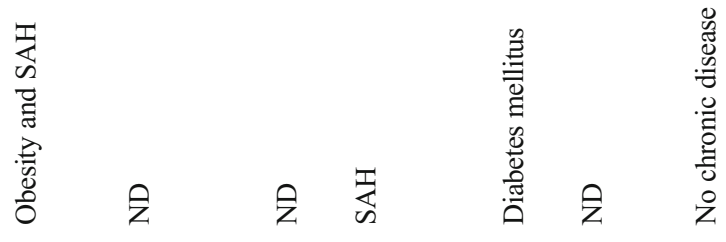

글

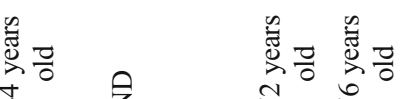

离

盾

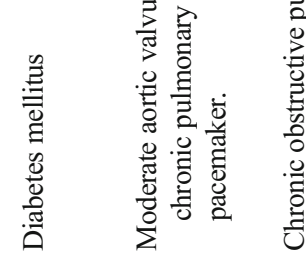

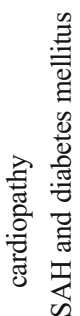

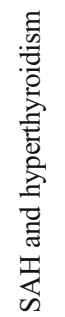

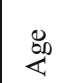

方

产

武

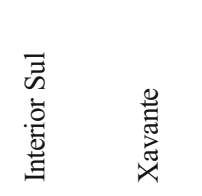

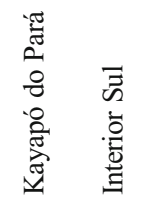

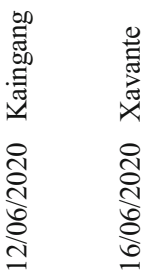

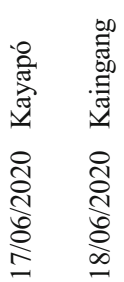

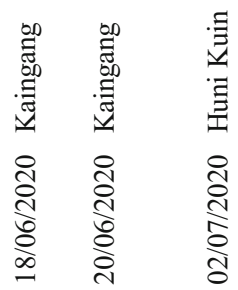

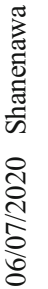

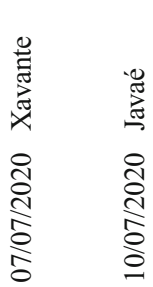

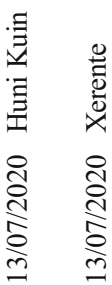

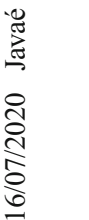




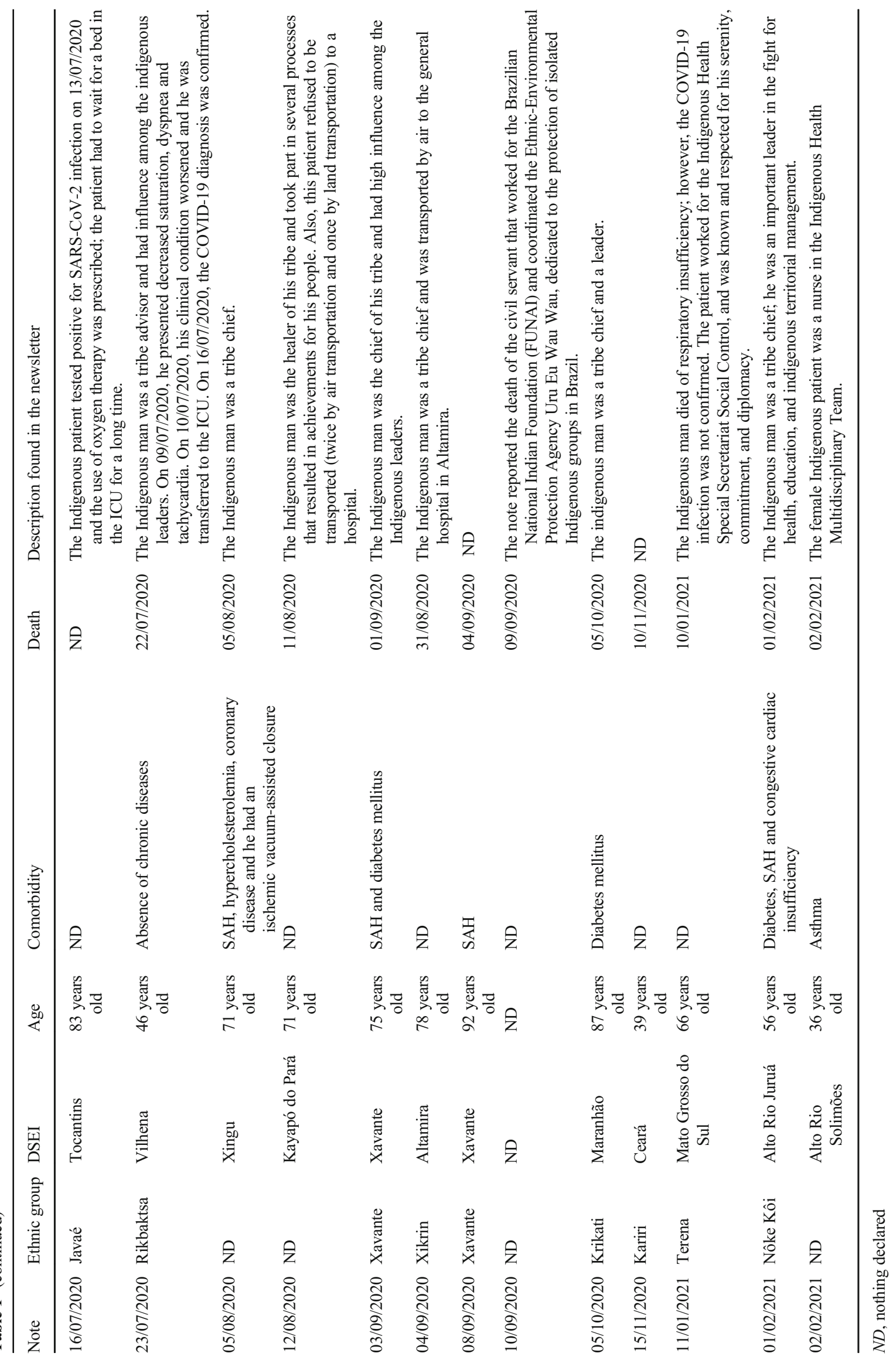


(Individuals between 50 and 59 years old) seven releases; death was reported in all of them. One of the patients was a tribe chief that presented comorbidities such as DM and SAH and congestive cardiac insufficiency. A report was found that one of the patient's family did not allow his transfer to a health service with more specialized assistance;

(Individuals between 60 and 69 years old) eleven releases; 10 of them reported death. The comorbidities listed were DM (three patients) and obesity (one patient) and one of the patients was recovering from arboviruses infection (dengue and yellow fever). One of the dead patients was a tribe chief. Three patients did not accept to be transferred to a health center with more specialized service. In one of the reports, the patient died of respiratory insufficiency, but the SARS-CoV-2 contamination was not confirmed;

(Individuals between 70 and 79 years old) fifteen releases; 14 of them reported death. Four of the dead patients were tribe chiefs and one was a tribe healer. Regarding comorbidities, one patient presented cardiac disease, one had pulmonary disease, four had SAH, and two presented DM and SAH. The families of three patients did not allow their transportation to health centers with more specialized service. One patient required river transport and another air transport to reach proper medical assistance;

(Individuals between 80 and 89 years old) nine releases; death was reported in eight of them. As for comorbidities, one individual presented SAH and another had DM. One patient presented both SAH and DM;

(Individuals between 90 and 99 years old) four releases; all of them reported death. One of the female patients had strong influence among the tribe women and was a midwife. Regarding comorbidities, one patient had SAH, one presented $\mathrm{DM}$, and another had chronic obstructive pulmonary disease.

Four notes were also published whose reports did not inform the age of the patients. Two of them reported the death of Indigenous leaders, one of them was a female leader.

\section{Pandemic characterization in the Indigenous population according to the DSEls}

In the early February 2021, the Health Ministry declared that there were 738 suspected cases, 41,949 confirmed cases, 51,235 dismissed cases, 4,300 infected and active cases, 36,986 recovered cases, and 554 deaths related to the COVID-19 (last update: 03/02/2021, 17:00) (Table 2). The notes only reported 62 deaths, that is, only $11.19 \%$ of the total deaths. In addition, the frequency of the publications throughout the pandemic was noticed to reduce. In April/2020, fifteen releases were selected for this study, in May/2020, there were 27 releases; in June/2020, seventeen releases were found; in $\mathrm{July} / 2020$, nine releases were selected; in August/2020, there were only two releases related to the relevant theme; in September/2020, four releases were found; in October/2020, one release was selected; in November/2020, there was one release; in January/2021, one release was found; and, finally, in February/2021, there were two releases.

Additionally, Table 2 draws attention to the high number of cases in three DSEIs, these are the DSEIs in Mato Grosso do Sul (3,902 cases), Interior Sul (2,284 cases), and Alto do Rio Solimões (2,007 cases). The DSEI in Mato Grosso do Sul also presented the highest number of deaths of Indigenous patients related to the COVID-19 ( 83 cases), followed by the DSEIs in Xavanté and in Leste de Roraima, with 47 death cases each. The DSEI structure according to the base municipality (Federal Unit); general Indigenous population assisted, number of ethnic groups; number of tribes; number of CASAIs; number of states and municipalities covered by the Indigenous assistance; and number of base centers are described in detail in Table 3.

\section{Indigenous importance in the Brazilian vaccination program development}

In the national vaccination program, the Indigenous peoples who live on indigenous lands were included in the first phase and the vaccination overview is presented in Table 4.

\section{Discussion}

\section{Cultural and ethnic diversity among the Indigenous peoples}

In 1500 , the indigenous population in Brazil was estimated in three million people, while in 1957, it was around 70,000 . This reduction is the result of several processes faced by these groups, from violent episodes to the outbreak of several epidemics [12]. Currently, there are many laws to protect these peoples, and this, somehow fostered some increase in the Indigenous population. According to the 2010 census, carried out by the IBGE, the indigenous population corresponded to 817,963 inhabitants [15]. This large number includes 305 different ethnic groups that speak 274 different languages (FUNAI), characterizing Brazil as the Latin American country with the greatest diversity of Indigenous ethnic groups.

The ethnic diversity is characterized by a wide range of social and cultural manifestations. In general, Indigenous peoples have certain specific customs such as sharing domestic utensils and community accommodations. These practices, commonly adopted by the Indigenous groups, become hazardous in the COVID-19 pandemic context, since they favor the virus spread among the members of the same community. Another remarkable characteristic of the Indigenous peoples is the use of medicinal therapies based on native herbs, and even if this kind of therapy might have a beneficial effect on the 
treatment of many diseases [16], including appearing to have potential in the treatment of COVID-19 symptoms and simple cases $[17,18]$; they would hardly be recommended to treat severe cases of the disease, which require admission in the intensive care unit and orotracheal intubation.

In such context, Indigenous peoples should be considered a population at risk for the COVID-19. Additionally, it seems relevant to emphasize that the term "Indigenous population" is generic and represents several ethnic groups that might become extinct because of this virus. In addition, the elderly are known to present higher chances of complication and death in the COVID-19, and this age group plays a crucial role within the Indigenous communities; since they are the individuals that carry the knowledge and customs of that people, with the function of passing on their knowledge, mainly through orality [13].

\section{Difficulty of differentiated access to health centers among Indigenous groups living in different states}

After reading the Health Ministry notes, a lack of accessibility faced by the Indigenous populations to the health units was observed. Reports were found of Indigenous patients that could not be transported to places with resources to assist them properly due to deficiencies in the transport system. Also, for them to reach specialized health centers, river and air transportation was required, resulting in delayed medical assistance that might be associated to the mortality of these individuals, mainly the older ones.

Transportation is not always the main accessibility problem. For example, in many cases, the Indigenous patient or their family members refused the transportation to the health unit with higher degree of specialization. This fact might be related, mainly, to the native people's fear of being inserted in a social context different from that where they are used to living. Therefore, the commitment of the health team is necessary to insert the Indigenous patients and their families in a health and disease context, highlighting the importance of treatment and follow-up in units with more complex treatments, mainly in more severe cases, with the presence of comorbidities and/or for affected individuals that belong to older age groups (the elderly).

Whenever possible, the health professionals must guarantee that the Indigenous patients do not abandon their culture; in cases when the use of more modern treatment is required, the health professional should be aware of the Indigenous culture and try to combine as much as possible the modern therapy to that used in the tribe. As a result, the Indigenous patient might feel welcomed and probably the refusal to more modern and effective treatments can be reduced.

\section{Factors associated to the COVID-19 pandemic spread in the Indigenous population}

According to Cupertino et al. (2020), with data obtained from the Health Ministry, on 15 May, there were 340 confirmed cases and 21 deaths in the Indigenous population [12]. On March 02, 2021, the number of confirmed cases was 41,855 and there were 549 deaths, showing a fast increase in the disease within this ethnic group. This fast advancement of the disease can be considered multifactorial. Being aware that the SARS-CoV-2 transmission is carried out mainly through interpersonal contact, one important factor of this increase in cases among the Indigenous population might be the Indigenous group proximity with other ethnic groups. The contact might occur through several activities such as tourism, wood exploitation, illegal hunting, drug dealing, missionary actions, and the return of Indigenous individuals from their workplace to the community, among others $[5,19]$.

Many other factors such as sharing domestic and cooking utensils and also the fact that a large number of Indigenous individuals share the same household might have contributed to the SARS-CoV-2 spread; however, these factors are the Indigenous population sociocultural characteristics, which makes it harder to prevent their occurrence. Even so, it is of great importance that the Brazilian Federal Government, along with the State governments and FUNAI, assist all indigenous populations that need it, by being the most transparent possible regarding the forms of infection by SARS-CoV-2. Simultaneously, information clarifying what the best prevention method is in each community is needed, in such a way that would never impose any sociocultural change, but rather by dialoguing with the Indigenous leaders in order to provide them with information about the best practices and respecting their sociocultural characteristics.

\section{Comorbidities in the Indigenous population and treatment refusal}

Belonging to the Indigenous population is a risk factor for respiratory diseases. In children under 1 year old, greater mortality was observed due to acute respiratory infections when compared to the general population [20]. Moreover, during the H1N1 pandemic in 2009, the incidence of severe respiratory condition was seen to be 4.5 times higher in the indigenous population when comparing to the rest of the Brazilian population [21]. Finally, factors such as obesity, SAH, and DM are risk factors for severe cases and higher risk of mortality by the COVID-19 [22] and all these conditions are found in the Indigenous population. According to the study put forward by Freitas et al. (2020) in the Jaguapiru tribe, the prevalence of DM was $4.5 \%$, obesity was $14.2 \%$ among male individuals and $30.8 \%$ in female individuals, and $\mathrm{SAH}$ 
Table 2 SARS-CoV-2 infection in Brazilian Indigenous people epidemiological data ${ }^{*}$

\begin{tabular}{|c|c|c|c|c|c|c|}
\hline DSEIs & Suspected & Confirmed & Dismissed & Infected (Active) & Clinical cure & Deaths \\
\hline Alagoas and Sergipe & 55 & 258 & 442 & 11 & 241 & 4 \\
\hline Altamira & 0 & 1,275 & 1,527 & 50 & 1,222 & 2 \\
\hline Alto Rio Juruá & 0 & 851 & 245 & 24 & 816 & 10 \\
\hline Alto Rio Negro & 34 & 2,152 & 881 & 129 & 2,001 & 20 \\
\hline Alto Rio Purus & 0 & 600 & 357 & 0 & 594 & 5 \\
\hline Alto Rio Solimões & 1 & 2,062 & 1,099 & 12 & 2,007 & 37 \\
\hline Amapá e Norte do Pará & 25 & 973 & 842 & 36 & 930 & 5 \\
\hline Araguaia & 0 & 344 & 539 & 21 & 316 & 7 \\
\hline Bahia & 15 & 718 & 1,046 & 66 & 645 & 7 \\
\hline Ceará & 30 & 966 & 1,738 & 45 & 911 & 8 \\
\hline Cuiabá & 31 & 1,301 & 771 & 56 & 1,221 & 24 \\
\hline Guamá-Tocantins & 4 & 1,489 & 2,033 & 20 & 1,450 & 17 \\
\hline Interior Sul & 47 & 2,462 & 4,015 & 135 & 2,284 & 41 \\
\hline Kaiapó do Mato Grosso & 7 & 985 & 1,029 & 6 & 973 & 5 \\
\hline Kaiapó do Pará & 31 & 1,213 & 1,162 & 3 & 1,174 & 9 \\
\hline Leste de Roraima & 18 & 3,748 & 3,834 & 1,974 & 1,720 & 47 \\
\hline Litoral Sul & 8 & 1,184 & 2,051 & 76 & 1,091 & 15 \\
\hline Manaus & 17 & 925 & 1,358 & 79 & 823 & 14 \\
\hline Maranhão & 0 & 1,676 & 1,042 & 1 & 1,643 & 27 \\
\hline Mato Grosso do Sul & 8 & 4,100 & 9,893 & 108 & 3,902 & 83 \\
\hline Médio Rio Purus & 0 & 506 & 15 & 0 & 501 & 5 \\
\hline Médio Rio Solimões and affluents & 6 & 752 & 953 & 14 & 725 & 11 \\
\hline Minas Gerais and Espírito Santo & 60 & 442 & 1,424 & 20 & 417 & 4 \\
\hline Parintins & 45 & 569 & 831 & 11 & 544 & 12 \\
\hline Pernambuco & 2 & 588 & 1,405 & 6 & 570 & 9 \\
\hline Porto Velho & 19 & 1,266 & 1,288 & 121 & 1,134 & 10 \\
\hline Potiguara & 7 & 675 & 860 & 2 & 669 & 4 \\
\hline Rio Tapajós & 0 & 1,959 & 2,437 & 47 & 1,895 & 12 \\
\hline Tocantins & 26 & 1,165 & 875 & 28 & 1,122 & 10 \\
\hline Vale do Javari & 0 & 821 & 364 & 6 & 811 & 2 \\
\hline Vilhena & 80 & 819 & 1,124 & 48 & 755 & 15 \\
\hline Xavante & 3 & 899 & 2,525 & 20 & 826 & 47 \\
\hline Xingu & 129 & 892 & 810 & 303 & 573 & 16 \\
\hline Yanomami & 30 & 1,314 & 420 & 822 & 480 & 10 \\
\hline Total & 738 & 41,949 & 51,235 & 4,300 & 36,986 & 554 \\
\hline
\end{tabular}

*Data updated up to the first week of February 2021

appeared in $29.7 \%$ in both sexes [23]. Curiously, the prevalence of such comorbidities differs from the national mean, since the self-reported DM in Brazil was around 6.9\% in 2013, and the SAH mean varied from 21.4 to $32.3 \%$, depending on the evaluation method used, while obesity was $20.7 \%$ in women and $18.7 \%$ in men [24, 25], which is probably directly related to the diet of the tribes.

Evaluating the different prevalence of comorbidities in Indigenous tribes is important, since each comorbidities shows a different degree of severity for the COVID-19 [26], which requires different measures in each tribe to decrease the chance of those individuals developing more severe conditions.

\section{How the SARS-CoV-2 spread among Indigenous peo- ples could have been prevented}

The Indigenous population is a risk group for the COVID19 [9]. Therefore, the adoption of practices to prevent this virus from spreading within the tribes is essential. Thus, restricting transportation of foreigners inside Indigenous areas is necessary, for example, by interrupting 
Table 3 Overview of the vaccination in Brazil-PHASE 1 and the Indigenous importance in the public health management

\begin{tabular}{|c|c|c|c|c|c|}
\hline $\begin{array}{l}\text { States and federal } \\
\text { district }^{*}\end{array}$ & $\begin{array}{l}60 \text {-year-old or over } \\
\text { institutionalized people }\end{array}$ & $\begin{array}{l}\text { Institutionalized people } \\
\text { with disabilities }\end{array}$ & $\begin{array}{l}\text { Indigenous peoples living on } \\
\text { indigenous land }\end{array}$ & $\begin{array}{l}34 \% \text { health } \\
\text { workers }\end{array}$ & $\begin{array}{l}\text { Target } \\
\text { population-phase } \\
1\end{array}$ \\
\hline Acre (AC) & 244 & 0 & 12,815 & 6,343 & 19,402 \\
\hline Amapá (AP) & 76 & 0 & 7,616 & 7,057 & 14,749 \\
\hline Amazonas (AM) & 400 & 60 & 101,156 & 32,813 & 134,429 \\
\hline Pará (PA) & 962 & 10 & 23,184 & 58,334 & 82,49 \\
\hline Rondônia (RO) & 140 & 0 & 7,784 & 15,595 & 23,519 \\
\hline Roraima (RR) & 100 & 0 & 36,834 & 4,833 & 41,767 \\
\hline Tocantins (TO) & 424 & 0 & 6,749 & 13,803 & 20,976 \\
\hline Northern region & 2,346 & 70 & 196,138 & 138,778 & 337,332 \\
\hline Alagoas (AL) & 1,246 & 10 & 7,946 & 32,594 & 41,796 \\
\hline Bahia (BA) & 9,788 & 285 & 27,201 & 142,087 & 179,361 \\
\hline Ceará (CE) & 2,398 & 132 & 20,250 & 86,380 & 109,160 \\
\hline Maranhão (MA) & 264 & 110 & 19,626 & 58,223 & 78,223 \\
\hline Paraíba (PR) & 1,212 & 120 & 10,432 & 42,925 & 54,689 \\
\hline Pernambuco (PE) & 2,462 & 130 & 26,506 & 99,924 & 129,022 \\
\hline Piauí (PI) & 460 & 10 & 21 & 28,651 & 29,142 \\
\hline $\begin{array}{l}\text { Rio Grande do } \\
\text { Norte (RN) }\end{array}$ & 1,400 & 10 & 0 & 37,848 & 39,258 \\
\hline Sergipe (SE) & 240 & 22 & 250 & 22,760 & 23,272 \\
\hline $\begin{array}{l}\text { Northeastern } \\
\text { region }\end{array}$ & 19,470 & 829 & 112,232 & 551,392 & 683,923 \\
\hline $\begin{array}{l}\text { Espírito Santo } \\
\text { (ES) }\end{array}$ & 2,970 & 210 & 2,793 & 42,273 & 48,246 \\
\hline $\begin{array}{l}\text { Minas Gerais } \\
\text { (MG) }\end{array}$ & 38,578 & 1,160 & 7,878 & 227,472 & 275,088 \\
\hline $\begin{array}{l}\text { Rio de Janeiro } \\
\text { (RJ) }\end{array}$ & 10,892 & 783 & 381 & 220,495 & 232,551 \\
\hline São Paulo (SP) & 42,604 & 1,357 & 3,727 & 598,518 & 646,206 \\
\hline $\begin{array}{l}\text { Southeastern } \\
\text { region }\end{array}$ & 95,044 & 3,510 & 14,779 & $1,088,758$ & $1,202,091$ \\
\hline Paraná (PR) & 12,224 & 482 & 10,816 & 102,959 & 126,481 \\
\hline $\begin{array}{l}\text { Rio Grande do Sul } \\
\text { (RS) }\end{array}$ & 9,510 & 380 & 14,348 & 138,523 & 162,761 \\
\hline $\begin{array}{l}\text { Santa Catarina } \\
\text { (SC) }\end{array}$ & 3,460 & 263 & 8,317 & 56,540 & 68,580 \\
\hline Southern region & 25,194 & 1,125 & 33,481 & 298,022 & 357,822 \\
\hline $\begin{array}{l}\text { Federal District } \\
\text { (FD) }\end{array}$ & 648 & 178 & 95 & 49,629 & 50,550 \\
\hline Goiás (GO) & 8,828 & 475 & 320 & 77,549 & 87,172 \\
\hline Mato Grosso (MT) & 2,382 & 190 & 28,758 & 28,744 & 60,074 \\
\hline $\begin{array}{l}\text { Mato Grosso do } \\
\text { Sul (MS) }\end{array}$ & 2,966 & 95 & 46,180 & 26,356 & 75,597 \\
\hline $\begin{array}{l}\text { Center-Western } \\
\text { region }\end{array}$ & 14,824 & 938 & 75,353 & 182,278 & 273,393 \\
\hline
\end{tabular}

missionary actions [13]. Additionally, it is relevant to reinforce the need for social isolation, use of masks, and gel alcohol. This might be a problem, since the adoption of such practices might be interpreted by the Indigenous individuals as loss of their cultural identity, possibly, damaging their mental health [27]. Moreover, although social isolation, use of masks, and gel alcohol and other attitudes are considered a public health measure by the World Health Organization to reduce the contagion rate, several speeches by the Brazilian President Jair Messias Bolsonaro are against this recommendation because he prioritizes the economy. It is a contradictory attitude that 
Table 4 Description of the Indigenous Health Special Districts in Brazil

\begin{tabular}{|c|c|c|c|c|c|c|c|c|}
\hline DSEI & $\begin{array}{l}\text { Base } \\
\text { Municipality } \\
\text { (FU) }\end{array}$ & $\begin{array}{l}\text { General indigenous } \\
\text { population assisted }\end{array}$ & $\begin{array}{l}\text { Ethnic } \\
\text { group }\end{array}$ & Tribe & CASAI & States covered & $\begin{array}{l}\text { Municipalities } \\
\text { covered }\end{array}$ & $\begin{array}{l}\text { Base } \\
\text { Center }\end{array}$ \\
\hline Alagoas Sergipe & Maceió (AL) & 12,250 & 12 & 31 & 0 & Alagoas and Sergipe & 10 & ND \\
\hline Altamira & Altamira (PA) & 3,974 & 10 & 60 & 1 & Pará & 5 & 1 \\
\hline Alto Rio Negro & $\begin{array}{l}\text { São Gabriel da } \\
\text { Cachoeira } \\
\text { (AM) }\end{array}$ & 40,233 & 25 & 707 & 1 & Amazonas & 3 & 25 \\
\hline Alto Rio Juruá & $\begin{array}{l}\text { Cruzeiro do Sul } \\
\text { (AC) }\end{array}$ & 17,672 & 17 & 148 & 1 & Acre & 8 & 7 \\
\hline Alto Rio Purus & $\begin{array}{l}\text { Rio Branco } \\
\text { (AC) }\end{array}$ & 13,555 & 7 & 150 & 1 & Amazonas, Acre, and Rondônia & 7 & 6 \\
\hline $\begin{array}{l}\text { Alto Rio } \\
\text { Solimões }\end{array}$ & Tabatinga (AM) & 70,519 & 7 & 234 & 1 & Amazonas & 7 & 12 \\
\hline $\begin{array}{l}\text { Alto Amapá and } \\
\text { northern Pará }\end{array}$ & Macapá (AP) & 12,440 & 11 & 139 & 2 & Amapá and Pará & 4 & 6 \\
\hline Araguaia & $\begin{array}{l}\text { São Félix do } \\
\text { Araguaia } \\
\text { (MT) }\end{array}$ & 5,562 & 8 & 41 & 1 & Mato grosso, Goiás, and Tocantins & 12 & 4 \\
\hline Bahia & Salvador (BA) & 29,284 & 21 & 77 & 0 & Bahia & 23 & 9 \\
\hline Ceará & Fortaleza (CE) & 26,129 & 15 & 100 & 1 & Ceará & 16 & 9 \\
\hline Cuiabá & Cuiabá (MT) & 6,830 & 10 & 120 & 3 & Mato grosso & 16 & 3 \\
\hline Guamá-Tocantins & Belém (PA) & 13,913 & 138 & 153 & 5 & Tocantins and Pará & 17 & 8 \\
\hline Interior Sul & $\begin{array}{l}\text { Florianópolis } \\
\text { (SC) }\end{array}$ & 63,118 & 4 & 180 & 0 & $\begin{array}{l}\text { São Paulo, Santa Catarina, and Rio } \\
\text { Grande do Sul }\end{array}$ & 65 & 8 \\
\hline Kaiapó MT & Colíder (MT) & 6,424 & 4 & 51 & 3 & Pará and Mato Grosso & 6 & 3 \\
\hline Kaiapó do Pará & Redenção (PA) & 5,796 & 1 & 50 & 4 & Pará & 6 & 4 \\
\hline Leste Roraima & Boa Vista (RR) & 53,213 & 7 & 323 & 0 & Roraima & 10 & 34 \\
\hline Litoral Sul & Curitiba (PR) & 22,975 & 11 & 129 & 2 & $\begin{array}{l}\text { São Paulo, Santa Catarina, Rio Grande } \\
\text { do Sul, Rio de Janeiro, and Paraná }\end{array}$ & 68 & 15 \\
\hline Manaus & Manaus (AM) & 30,768 & 35 & 218 & 1 & Amazonas & 15 & 16 \\
\hline Maranhão & São Luís (MA) & 36,060 & 8 & 424 & 3 & Maranhão & 16 & 6 \\
\hline $\begin{array}{l}\text { Mato Grosso do } \\
\text { Sul }\end{array}$ & $\begin{array}{l}\text { Campo Grande } \\
\text { (MS) }\end{array}$ & 83,434 & 8 & 99 & 3 & Mato Grosso do Sul & 129 & 15 \\
\hline Médio Rio Purus & Lábrea (AM) & 6,822 & 17 & 105 & 2 & Amazonas & 3 & 10 \\
\hline $\begin{array}{l}\text { Médio Rio } \\
\text { Solimões and } \\
\text { affluents }\end{array}$ & Tefé (AM) & 24,538 & 16 & 184 & 2 & Amazonas & 14 & 15 \\
\hline $\begin{array}{l}\text { Minas Gerais and } \\
\text { Espírito Santo }\end{array}$ & $\begin{array}{l}\text { Governador } \\
\text { Valadares } \\
\text { (MG) }\end{array}$ & 16,648 & 10 & 93 & 2 & Minas Gerais and Espírito Santo & 14 & 18 \\
\hline Parintins & Parintins (AM) & 16,911 & 2 & 124 & 2 & Amazonas and Pará & 5 & 13 \\
\hline Pernambuco & Recife (PE) & 39,231 & 13 & 224 & 1 & Pernambuco & 15 & 12 \\
\hline Porto Velho & $\begin{array}{l}\text { Porto Velho } \\
\text { (RO) }\end{array}$ & 10,311 & 68 & 172 & 6 & $\begin{array}{l}\text { Rondônia, Mato Grosso, and } \\
\text { Amazonas }\end{array}$ & 15 & 5 \\
\hline Pontiguara & $\begin{array}{l}\text { João Pessoa } \\
\text { (PB) }\end{array}$ & 14,024 & 1 & 33 & 0 & Paraíba & 3 & 3 \\
\hline Rio Tapajós. & Itaituba (PA) & 12,722 & 4 & 141 & 4 & Pará & 4 & 11 \\
\hline Tocantins & Palmas (TO) & 11,908 & 11 & 160 & 2 & Tocantins and Goiás & 12 & 5 \\
\hline Vale do Javari & $\begin{array}{l}\text { Atalaia do Norte } \\
\text { (AM) }\end{array}$ & 6,263 & 6 & 59 & 1 & Amazonas & 1 & 8 \\
\hline Vilhena & Cacoal (RO) & 7,159 & 17 & 172 & 4 & Amazonas & 2 & 4 \\
\hline Xavante & $\begin{array}{l}\text { Barra do Garças } \\
\text { (MT) }\end{array}$ & 20,653 & 1 & 305 & 1 & Mato Grosso & 12 & 6 \\
\hline Xingu & Canarana (MT) & 7,213 & 16 & 81 & 4 & Mato Grosso & 8 & 4 \\
\hline Yanomami & Boa Vista (RR) & 25,486 & 2 & 323 & 1 & Roraima and Amazonas & 1 & 37 \\
\hline
\end{tabular}

$N D$, nothing declared; CASAI, Indigenous health houses; DSEI, Indigenous Health Special Districts; FU, federation unit 
can cause a higher number of COVID-19 cases and deaths prompting the population to higher contagion rate with no or limited health supported. This fact can be worst among Indigenous peoples with a low access for health services. Moreover, the Federal Government neglects the Indigenous as a vulnerable group for the COVID-19 pandemic [9]. Because the Indigenous population is a risk group for the COVID-19, they were included in the Brazilian vaccination program as a priority group [28]. This measure is very important, since the control of the virus spread and the immunity of the elderly are fundamental for the preservation of the Indigenous culture diversity.

\section{Other ethnic minority groups and people living on more deprived regions}

Brazil is a country with a heterogeneous population, so there are other ethnic minorities besides Indigenous people and other people living on more deprived regions, such as Brown and Blacks. These people can be more susceptible for mortality due to COVID-19 as shown in a study that comprised 11,321 individuals with a positive test RT-PCR for SARS-COV-2 considering ethnic and racial issues on Brazil [29]. Curiously, a higher mortality in Brown and Black individuals comparing to White people was demonstrated [29]. Another interesting fact was the higher hazard ratios for mortality for the people from the northern and northeast regions from Brazil where the access for health services is low [29]. Baqui et al. (2020) demonstrated the outcomes for eight Indigenous according to COVID-19, being described five non-survivors [29]. The North region is the Brazilian region with the largest number of Indigenous people according FUNAI.

The divergence of prognosis to COVID-19 in different ethnicities has been noticed in other countries as well. Millett et al. (2020) conducted a study about American Black population, and it was noticed that cities with a higher concentration of Black people were associated with higher numbers of COVID-19 cases, as well as higher mortality [30]. In the cities with great counties of Black people, also were observed a higher number of comorbidities, elderly people $\geq 65$ years of age, unemployment, lack of health insurance, and a higher air pollution index [30]. In brief, it was demonstrated that among the $\sim 20 \%$ US counties are disproportionately Black, and they accounted for 52\% of COVID-19 diagnoses and $58 \%$ of COVID-19 deaths in the USA [30]. Curiously, the same outcome occurred at Baqui et al. on Brazil [29].

Holtgrave et al. also noticed differences of prognosis between white non-Hispanic, Black non-Hispanic, and Hispanic adults in New York City [31]. The mortality risk from COVID-19 in non-Hispanic Black adults and Hispanics was 5.38 and 3.48 times greater, respectively, than non-Hispanic Whites. It is known that the Black population is considered vulnerable, considering that they correspond to an ethnic group with a higher poverty rate and, consequently, less access to health services [32]. The divergence was also noticed in the city of Chicago, and in the states of Louisiana and Michigan [33]. In Chicago and Louisiana, the Black people correspond to $\sim 30 \%$ of the population, and $\sim 70 \%$ of reported deaths due to COVID19. In Michigan, Black people constitute $14 \%$ of the population and represent $\sim 40 \%$ of deaths due COVID-19 [33]. As discussed by Holtgrave et al., Hispanic had differences in infection experience and non-Hispanic Black had differences in both infection experience and in the need for hospitalization due the SARS-COV-2, relative to White to be the cause for the disparities [31].

Also, Lusignan et al. analyzed a population sample from Oxford and noted that the chances of contamination rate by SARS-CoV-2 were higher in people of other ethnicity than White or in people living in poorer areas [34]. The problem behind this situation extends beyond the health issue, also affecting economic issues, considering that the measures of social restriction to combat the COVID-19 pandemic cause huge economic impact, mainly among the people who deserve more attention, due to higher social and economic vulnerability.

Once the social vulnerabilities are identified, it is important to discuss the epidemiological aspects that involve the different minority groups and people living on more deprived regions to implement a better planning to combat the pandemic and to preserve each group. However, it was not the reality. Unfortunately, in state of Connecticut, for example, there was negligence in the racial characterization of the victims of COVID-19: more than 50\% of the laboratory reports related to the disease in question did not contain information about race [32].

Brazil should implement measures to deal with the pandemic considering the vulnerability groups related to COVID-19 being the Indigenous peoples the most vulnerable to infection with SARS-CoV-2 and the most impoverished monetarily [35]. Furthermore, Brown and Black people are more vulnerable to infection with SARS-CoV-2 than White and Asian groups, and the states with greatest vulnerability deserve more attention to control the pandemic [35].

Unfortunately, the negligence of minority groups and people living on more deprived regions is not a current problem. For example, it was also noted the different impacts during the H1N1 pandemic among minority groups and people living on more deprived regions. A study done in England during 2009 and 2010 outbreak analyzed the deaths due to H1N1. It was observed that $19.9 \%$ of deaths corresponded to non-white ethnicities, while this population corresponds to only $12.5 \%$ of the country population [36]. 


\section{Limitations}

There were limitations in the data collection from the Brazilian Health Ministry database. Several published releases (notes) did not present complete information, and in many of the information such as DSEI of origin, ethnic group, age, or the presence of comorbidities was not reported. Additionally, some negligence was observed regarding the publication of information during the pandemic. In May, for example, twenty-seven notes described the criteria determined for inclusion in this paper, while in other months, only one newsletter met the inclusion requirements.

\section{Conclusion}

Taking into consideration the concept of universality, the Indigenous population must be assisted in a holistic way, considering their customs and traditions. Therefore, more specialized health units should be made available closer to the tribes to provide proper assistance to this population during the current pandemic and in future emerging problems that might affect that population.

Code availability Not applicable.

Author contribution All authors have approved the manuscript and agreed with its submission to the journal. Also, all authors wrote and revised the manuscript.

Data availability Not applicable.

\section{Declarations}

Ethics approval and consent to participate Not applicable.

Consent for publication Not applicable.

Additional declarations for articles in life science journals that report the results of studies involving humans and/or animals Not applicable.

Competing interests The authors declare no competing interests.

\section{References}

1. World Health Organization. 2020. Available in https://www.who. int/dg/speeches/detail/who-director-general-s-opening-remarks-atthe-media-briefing-on-covid-19-11-march-2020. Accessed 21 Aug 2020.

2. Campos DMO, Fulco UL, de Oliveira CBS, Oliveira JIN. SARSCoV-2 virus infection: targets and antiviral pharmacological strategies. J Evid Based Med. 2020;13(4):255-60. https://doi.org/10. 1111/jebm.12414.
3. Funai - Brasil. Available in http://www.funai.gov.br/index.php/ indios-no-brasil/quem-sao. Accessed 09 Feb 2021.

4. Rocha DFD, Porto MFS, Pacheco T. A luta dos povos indígenas por saúde em contextos de conflitos ambientais no Brasil (19992014) [The struggle of indigenous peoples for health in environmental conflict contexts in Brazil (1999-2014)]. Cien Saude Colet. 2019;24(2):383-92.

5. Palamim CVC, Ortega MM, Marson FAL. COVID-19 in the Indigenous population of Brazil. J Racial Ethn Health Disparities. 2020;7(6):1053-8. https://doi.org/10.1007/s40615-020-00885-6.

6. Carino G, Diniz D. Deforestation and Brazil's Indigenous population. Lancet. 2019;394(10216):2241. https://doi.org/10.1016/ S0140-6736(19)32620-0.

7. Ferrante L, Fearnside PM. Brazil threatens Indigenous lands. Science. 2020;368(6490):481-2. https://doi.org/10.1126/science. abb6327.

8. Ferrante L, Fearnside PM. Military forces and COVID-19 as smokescreens for Amazon destruction and violation of indigenous rights. ERDE. 2020;151:258-63. https://doi.org/10.12854/erde2020-542.

9. Ferrante L, Fearnside PM. Protect Indigenous peoples from COVID-19. Science. 2020;368:251-25251. https://doi.org/10. 1126/science.abc0073.

10. Cardoso MD. Health and indigenous peoples in Brazil: notes on some current policy mistakes. Cad Saúde Públ. 2014;30(4):860-6. https://doi.org/10.1590/0102-311x00027814.

11. Langdon EJ, Garnelo L. Articulation between health services and "indigenous medicine": anthropological reflections on policies and reality in Brazil. Salud Colect. 2017;13(3):457-70. https://doi.org/ 10.18294/sc.2017.1117.

12. Cupertino GA, Cupertino MDC, Gomes AP, Braga LM, SiqueiraBatista R. COVID-19 and Brazilian Indigenous populations. Am J Trop Med Hyg. 2020;103(2):609-12. https://doi.org/10.4269/ ajtmh.20-0563.

13. Ferrante L, Steinmetz WA, Almeida ACL, Leão J, Vassão RC, Tupinambás U, et al. Brazil's policies condemn Amazonia to a second wave of COVID-19. Nat Med. 2020;26(9):1315. https:// doi.org/10.1038/s41591-020-1026-x.

14. Saúde Indígena. Ministério da Saúde do Brasil. Available in https:// saudeindigena1.websiteseguro.com/coronavirus/notas.php. Accessed 09 Feb 2021.

15. Instituo Brasileiro de Geografia e Estatística. Características gerais da população, religião e pessoas com deficiência. Rio de Janeiro: IBGE; 2010. Available in https://censo2010.ibge.gov.br. Accessed 15 Dec 2020

16. Ernst E. The efficacy of herbal medicine-an overview. Fundam Clin Pharmacol. 2005;19(4):405-9. https://doi.org/10.1111/j. 1472-8206.2005.00335.x.

17. Silveira D, Prieto-Garcia JM, Boylan F, Estrada O, Fonseca-Bazzo YM, Jamal CM, et al. COVID-19: is there evidence for the use of herbal medicines as adjuvant symptomatic therapy? Front Pharmacol. 2020;11:581840. https://doi.org/10.3389/fphar.2020. 581840 .

18. de Abreu DJM, de Barros HEA, Natarelli CVL, Zanzini AP, de Boas EVBV, Piccoli RH, et al. Therapeutic approach for COVID19: the power of herbal medicine. RSD. 2020;9(10):e6789108907. https://doi.org/10.33448/rsd-v9i10.8907.

19. Simionatto S, Barbosa M, Marchioro SB. COVID-19 in Brazilian indigenous people: a new threat to old problems. Soc Bras Med Trop. 2020;53:e20200476. https://doi.org/10.1590/0037-86820476-2020.

20. Ministério da Saúde, Secretaria de Vigilância em Saúde, Departamento de Vigilância de Doenças e Agravos não transmissíveis e Promoção da Saúde. VIGITEL Brasil 2013: Vigilância de fatores de risco e proteção para doenças crônicas por inquérito telefônico. 2014. Accessed on 2 January 2021. 
Available in http://www.prefeitura.sp.gov.br/cidade/secretarias/ upload/saude/arquivos/morbidade/Vigitel-2013.pdf.

21. La Ruche G, Tarantola A, Barboza P, Vaillant L, Gueguen J, Gastellu-Etchegorry M, et al. The 2009 pandemic H1N1 influenza and indigenous populations of the Americas and the Pacific. Euro Surveill. 2009;14(42):19366. https://doi.org/10.2807/ese.14.42. 19366-en.

22. Jordan RE, Adab P, Cheng KK. COVID-19: risk factors for severe disease and death. BMJ. 2020;368:m1198. https://doi.org/10.1136/ bmj.m1198.

23. Freitas GA, Souza MCC, Lima RC. Prevalência de diabetes mellitus e fatores associados em mulheres indígenas do Município de Dourados, Mato Grosso do Sul, Brasil. Cad Saúde Públ. 32(8):e00023915. https://doi.org/10.1590/0102$311 X 00023915$.

24. Malta DC, Gonçalves RPF, Machado IE, Freitas MIF, Azeredo C, Szwarcwald CL. Prevalência da hipertensão arterial segundo diferentes critérios diagnósticos, Pesquisa Nacional de Saúde. Rev Bras Epidemiol. 2018;21(1):e180021. https://doi.org/10.1590/ 1980-549720180021.supl.1.

25. National Cancer Institute. Brasileiros atingem maior índice de obesidade dos últimos treze anos, de acordo com pesquisa Vigitel. 2019. Accessed 2 Jan 2021. Available at https://www. inca.gov.br/en/node/3433\#: :text=No\%20Brasil\%2C\%20mais\% 20da\%20metade, $\% 2 \mathrm{C} \% 20$ com\%2055\%2C7\%25.

26. Yang J, Zheng Y, Gou X, Pu K, Chen Z, Guo Q, et al. Prevalence of comorbidities and its effects in patients infected with SARS-CoV-2: a systematic review and meta-analysis. Int J Infect Dis. 2020;94: 91-5. https://doi.org/10.1016/j.ijid.2020.03.017.

27. Júnior JG, Moreira MM, Pinheiro WR, de Amorim LM, Lima CKT, da Silva CGL, et al. The mental health of those whose rights have been taken away: an essay on the mental health of indigenous peoples in the face of the 2019 Coronavirus (2019-nCoV) outbreak. Psychiatry Res. 2020;289:113094.

28. Ministério da Saúde. Profissionais de saúde e idosos institucionalizados serão os primeiros a receber doses de vacina contra a Covid-19. 2021. Available in https://www.gov.br/saude/ pt-br/assuntos/noticias/profissionais-de-saude-e-idososinstitucionalizados-serao-os-primeiros-a-receber-doses-de-vacinacontra-a-covid-19. Accessed 09 Feb 2021.
29. Baqui P, Bica I, Marra V, Ercole A, Schaar MVD. Ethnic and regional variations in hospital mortality from COVID-19 in Brazil: a cross-sectional observational study. Lancet Glob Health. 2020;8(8):1018-26. https://doi.org/10.1016/s2214-109x(20) 30285-0.

30. Millett GA, Jones AT, Benkeser D, Baral S, Mercer L, Beyrer C, et al. Assessing differential impacts of COVID-19 on black communities. Ann Epidemiol. 2020;47:37-44. https://doi.org/10.1016/ j.annepidem.2020.05.003.

31. Holtgrave DR, Barranco MA, Tesoriero JM, Blog DS, Rosenberg ES. Assessing racial and ethnic disparities using a COVID-19 outcomes continuum for New York State. Ann Epidemiol. 2020;48:914. https://doi.org/10.1016/j.annepidem.2020.06.010.

32. Laurencin CT, McClinton A. The COVID-19 Pandemic: a call to action to identify and address racial and ethnic disparities. J Racial Ethn Health Disparities. 2020;7(3):398-402. https://doi.org/10. 1007/s40615-020-00756-0.

33. Yancy CW. COVID-19 and African Americans. JAMA. 2020;323(19):1891-2. https://doi.org/10.1001/jama.2020.6548.

34. de Lusignan S, Dorward J, Correa A, Jones N, Akinyemi O, Amirthalingam $\mathrm{G}$, et al. Risk factors for SARS-CoV-2 among patients in the Oxford Royal College of General Practitioners Research and Surveillance Centre primary care network: a crosssectional study. Lancet Infect Dis. 2020;20(9):1034-42. https://doi. org/10.1016/S1473-3099(20)30371-6.

35. Tavares FF, Betti G. The pandemic of poverty, vulnerability, and COVID-19: evidence from a fuzzy multidimensional analysis of deprivations in Brazil. World Dev. 2021;139:105307. Epidemiol Infect. 2015;143(16):3375-83. https://doi.org/10.1016/j.worlddev. 2020.105307.

36. Zhao H, Harris RJ, Ellis J, Pebody RG. Ethnicity, deprivation and mortality due to 2009 pandemic influenza A(H1N1) in England during the 2009/2010 pandemic and the first post-pandemic season. Epidemiol Infect. 2015;143(16):3375-83. https://doi.org/10.1017/ S0950268815000576.

Publisher's Note Springer Nature remains neutral with regard to jurisdictional claims in published maps and institutional affiliations. 